\title{
Wie gut kennen die Fachgesellschaften die Richtlinien «Zusammenarbeit Ärzteschaft- Industrie»?
}

\section{Hermann Amstad}

Dr. med., Generalsekretär der Schweizerischen Akademie der Medizinischen Wissenschaften, Basel

Korrespondenz:

Dr. med. Hermann Amstad

SAMW

Petersplatz 13

CH-6051 Basel

Fax 0612699039

mail@samw.ch
Tel. 0612699030

Die Richtlinien «Zusammenarbeit Ärzteschaft-Industrie», welche die Schweizerische Akademie der Medizinischen Wissenschaften (SAMW) Ende 2005 verabschiedet und die FMH 2006 in ihre Standesordnung aufgenommen hat, haben einen gewissen «Kulturwandel» bei der Ärzteschaft bewirkt. Die Beratende Kommission, welche die Umsetzung der Richtlinien begleitet, hat allerdings festgestellt, dass noch immer Anlässe als «Fortbildungsveranstaltungen» gelten, obwohl sie den Richtlinien der SAMW nicht genügen.

Zahlreiche Fachgesellschaften (und auch die Arbeitsgruppe «Unterstützung der Fachgesellschaften im Bereich der Fortbildung» der früheren KWFB) haben signalisiert, dass sie froh wären um eine Art Checkliste, mit der geprüft werden könnte, ob ein Fortbildungsanlass den Richtlinien entspricht. Die Beratende Kommission hat dieses Anliegen aufgegriffen und im Juni 2009 eine solche Checkliste veröffentlicht; sie ist auf der SAMW-Website online abrufbar.

Die Checkliste enthält eine erste Seite mit allen notwendigen Angaben zum geplanten Anlass sowie eine zweite Seite mit den zu erfüllenden Kriterien. Diese Checkliste ist vom Antragsteller bzw. der Antragstellerin auszufüllen und zusammen mit dem Antrag auf Credits bei der Fachgesellschaft einzureichen. Falls alle Fragen dieser Checkliste mit Ja beantwortet werden, bedeutet dies, dass der Anlass den Richtlinien entspricht.

Im Frühjahr 2010, d.h. vier Jahre nach Inkrafttreten der Richtlinien und etwas mehr als ein halbes Jahr nach der Veröffentlichung der Checkliste, schrieb die Beratende Kommission die Präsidentin-

\begin{tabular}{|c|c|}
\hline \multicolumn{2}{|c|}{$\begin{array}{l}\text { Kriterien der Checkliste, die bei der Anerkennung von Fortbildungsanlässen berücksichtigt } \\
\text { werden }(\mathrm{N}=35) \text { : }\end{array}$} \\
\hline Ärzte als Veranstalter & $31(89 \%)$ \\
\hline Inhalt unabhängig von Sponsoring & $33(94 \%)$ \\
\hline Rahmenprogramm untergeordnet & $30(86 \%)$ \\
\hline Kein Monosponsoring & $26(74 \%)$ \\
\hline Schriftliche Sponsoringvereinbarung & $21(60 \%)$ \\
\hline Kostenbeteiligung der Teilnehmer & $25(71 \%)$ \\
\hline Offenlegung von Interessenkonflikten & $24(69 \%)$ \\
\hline Weitere & $6(17 \%)$ \\
\hline Keine & 1 (3\%) \\
\hline
\end{tabular}

nen und Präsidenten aller 45 Fachgesellschaften der Schweiz an und fragte nach,

- ob sie die Richtlinien kennen,

- ob sie diese anwenden (und in welcher Form),

- ob die Umsetzung Probleme bereitet.

Diejenigen, die nach einem Monat noch nicht geantwortet hatten, wurden erneut (per E-Mail) angeschrieben.

\section{Kenntnis der Richtlinien und Beachtung der Checkliste}

Von 45 angeschriebenen Fachgesellschaften haben 35 (78\%) geantwortet. 32 der antwortenden Präsidentinnen bzw. Präsidenten (d.h. 91\%) kennen die Richtlinien persönlich. Bei 25 Fachgesellschaften (71\%) nimmt das Fortbildungsprogramm Bezug auf die Richtlinien.

Die Kriterien der Checkliste werden in mehr als der Hälfte der Fälle erfüllt (siehe Tab. 1): Am niedrigsten sind die Ja-Antworten mit $60 \%$ beim Kriterium «Schriftliche Vereinbarung», am höchsten (mit 94\%) beim Kriterium «Inhalt unabhängig vom Sponsoring». Lediglich eine Fachgesellschaft gibt an, dass sie bei der Anerkennung von Fortbildungsveranstaltungen keines der Kriterien berücksichtigt.

\section{Probleme bei der Umsetzung der Richtlinien}

Probleme bei der Umsetzung geben 12 (34\%) der antwortenden Fachgesellschaften an. Die Antworten hier waren teilweise sehr kritisch («Complication inutile de l'organisation des manifestions»). Sehr häufig genannt werden Probleme im Zusammenhang mit dem Monosponsoring. Nachfolgend findet sich dazu eine Auswahl von Zitaten aus den Rückmeldungen:

- «Probleme bestehen noch in regionalen Vereinen, Monosponsoring ist dort mehr Regel als Ausnahme (Fortbildung> mit Nachtessen, neudeutsch: Apéro riche), Sponsoring von Versammlungen örtlicher Vereine usw.»

- «Es kommt vor, dass wir ein Monosponsoring akzeptieren müssen, wenn uns das Thema und der Referent adäquat erscheinen.»

- «Einige Veranstaltungen haben Monosponsoring, dürfen aber trotzdem als «unabhängig` angesehen werden.»

- «Das geforderte Polysponsoring kann durch 〈Pseudopolysponsoring〉 mit Tochterfirmen usw. 
umgangen werden. Auf der andern Seite sehen wir durchaus Fälle von absolut korrektem, nicht korrumpiertem Monosponsoring.»

- «Ein weiterer Punkt ist die Frage des Monosponsorings in der heutigen Zeit. Als praktisches Beispiel sei hier die in Amerika börsenkotierte Firma XY genannt, es gibt weitere Beispiele. Die Gruppe $\mathrm{XY}$ besteht aus 15 verschiedenen Unternehmungen im Medizinalbereich. Jede ist finanziell eigenständig. Kann nun, wenn zwei oder drei Firmen aus der gleichen Gruppe eine Veranstaltung unterstützen, von Monosponsoring gesprochen werden, oder ist man hier tolerant und geht das Risiko eines verdeckten Monosponsorings ein?»

- «Eine weitere Möglichkeit ist, sich einen branchenfremden Sponsor auszusuchen, z.B. eine Firma, die Impfstoffe herstellt, nimmt sich als Co-Sponsor die Ärztekasse. Dass die Vorträge dann neutral gehalten werden, macht die Sache nicht sympathischer.»
- «Die Überprüfung ist in der Praxis schwierig, da die Angaben aus den Ausschreibungen häufig nicht hervorgehen und damit auch nicht überprüfbar sind.»

Und es gibt auch Fachgesellschaften, die keine Probleme haben - vielleicht, weil sie es sich (zu?) einfach machen: «Die Fachgesellschaft hat bisher darauf verzichtet, für Veranstaltungen irgendwelche Empfehlungen resp. Credits zu Handen ihrer Mitglieder herauszugeben, da sie davon ausgeht, dass die Mitglieder die Qualität einer Weiterbildungsveranstaltung selber beurteilen können.»

\section{Fazit}

Die hohe Rücklaufquote von fast $80 \%$ ist erfreulich; sie zeigt, dass - zumindest bei den Präsidentinnen und Präsidenten der Fachgesellschaften - eine gewisse Sensibilisierung für die Thematik vorhanden ist. Dieser Eindruck wird noch verstärkt durch die Tat-

\section{Die hohe Rücklaufquote von fast $\mathbf{8 0} \%$ zeigt, dass - zumindest bei den Präsidentinnen und Präsidenten der Fachgesellschaften - eine gewisse Sensibilisierung für die Thematik vorhanden ist}

- «Manchmal ist das Monosponsoring nicht zu vermeiden, da es in einem Bereich nur eine Firma gibt.»

Die Organisation kleiner Fortbildungsanlässe wird nach Ansicht einiger Fachgesellschaften erschwert; dazu zwei Zitate:

- «Wenn jeder Punkt berücksichtigt wird, wird die Organisation von «kleinen` Veranstaltungen (z. B. 1-2 Stunden Weiterbildung ausserhalb vom Kongress bzw. Symposium) so erschwert, dass es kontraproduktiv wirkt und auf eine punktuelle Weiterbildung verzichtet wird.»

- «Das Problem für kleinere Veranstaltungen ist, wenn die teilnehmenden Ärzte zahlen müssen. Der Aufwand wäre für viele von unseren Veranstaltungen einfach zu gross.»

Einige Fachgesellschaften betrachten die Richtlinien als schwer umsetzbar:

- «Eine Vereinbarung zu überprüfen, sprengt den Rahmen unserer Begutachtung. Dies soll zwischen Veranstalter und Sponsor geregelt werden. Unsere persönliche Meinung ist, dass hier eine Überregulierung besteht.» sache, dass die Kriterien der Checkliste von mehr als der Hälfte der Fachgesellschaften beachtet werden.

Die Beratende Kommission betrachtet die Rückmeldungen als wertvolle Denkanstösse; namentlich die Schwierigkeiten bei der Umsetzung der Richtlinien nimmt sie sehr ernst. Die Kommission wird sorgfältig prüfen, ob und welche Lösungen sich hier finden lassen. Erste Ansätze dazu sind bereits vorhanden: Auf der SAMW-Website (www.samw.ch $\rightarrow$ Ethik $\rightarrow$ Zusammenarbeit Ärzteschaft-Industrie) steht nicht nur die Checkliste, sondern seit kurzem auch eine Muster-Sponsoringvereinbarung zur Verfügung.

Weiterhin gilt, was die SAMW in der Präambel der Richtlinien «Zusammenarbeit Ärzteschaft-Industrie» festgehalten hat: «Indem die Ärzteschaft für sich selber Leitplanken formuliert, welche die bestehenden Vorschriften präzisieren und ergänzen, unterstreicht sie ihren Willen zur Unabhängigkeit und Glaubwürdigkeit. ... [Die Richtlinien] sollen nicht verbieten, sondern zur Förderung von Objektivität und Qualität, zur Transparenz, zur Vermeidung von Abhängigkeiten und zum bewussten Umgang mit Interessenkonflikten beitragen.» 